\title{
Femmes et Physique, la commission reprend du service
}

La commission « Femmes et Physique » de la Société Française de Physique a pour objectif d'attirer, d'encourager et de promouvoir les femmes dans les métiers de la physique ${ }^{(1)}$.

La proportion de femmes chercheuses, enseignantes-chercheuses ou ingénieures reste encore faible en France, même au niveau des candidatures ${ }^{(2)}$.

Cette démotivation pour la physique a de multiples causes. Pour attirer plus de filles dans nos métiers, il faut leur faire rencontrer des physiciennes accomplies et enthousiastes.

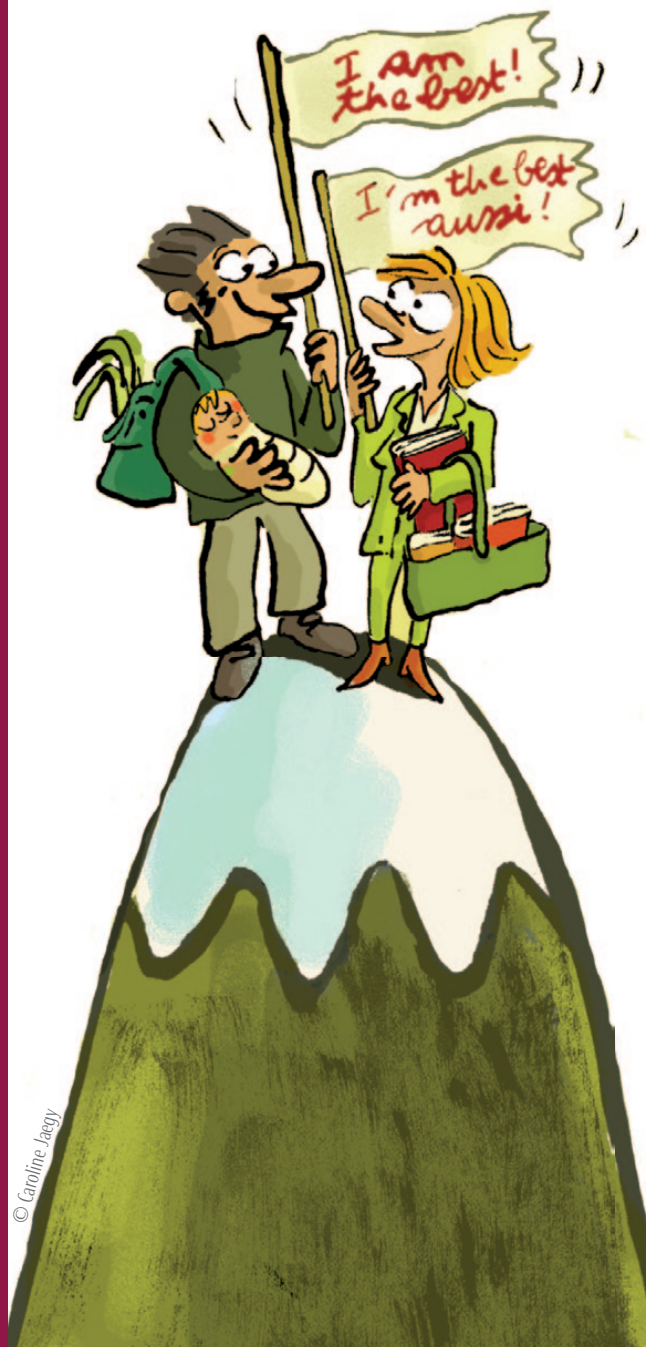

Il est vrai que les promotions, nominations à des postes de responsabilité, invitations à des congrès et nominations à des prix ${ }^{(3)}$ s'adressent encore majoritairement aux hommes. Cette constatation se vérifie dans toutes les disciplines scientifiques et dans tous les établissements, mais surtout en physique (voir tableau). Le "plafond de verre $"^{(4)}$, invisible mais résistant, qui empêche les femmes d'atteindre des postes élevés, est bien connu dans toutes les branches, mais il a un effet encore plus désastreux dans les disciplines réputées difficiles et abstraites comme la physique.

Pour tenter de corriger les facteurs à l'origine de cet état de fait - les femmes sont moins présentes dans les différents comités de sélection (embauche, promotions, financement, conférences), elles se mettent moins en avant, elles vont moins en congrès puisqu'elles sont plus souvent responsables de l'organisation matérielle au sein du couple, auprès des enfants ou de parents âgés -, nos actions seront les suivantes :

- Inciter les femmes de la SFP à donner des conférences dans les collèges et les lycées pour inciter les jeunes (filles et garçons) à faire de la physique (notamment dans le cadre des contacts existants avec les rectorats, universités et associations ${ }^{(1)}$ comme "Femmes et sciences").

- Mettre sur le site internet de la SFP une base de données de conférencières pour les différents types d'interventions : conférences de spécialistes, " grand public de physicien(ne)s ", " grand public " et "public de collégien(ne)s et lycéen(ne)s ".

- Augmenter la proportion de femmes dans les différents conseils et comités de sélection (SFP, CNRS, CNU, ANR, Europe...), en encourageant les femmes à se présenter aux élections ou à s'inscrire dans les bases de données, et en précisant sur le site de la commission «Femmes et Physique $»^{(5)}$ les modalités pour candidater. - Demander aux différentes instances de prendre plus largement en compte dans les critères de sélection pour les embauches, les prix ou les promotions, les travaux de vulgarisation (vers les scolaires ou le grand public), les actions au service de la collectivité, etc., souvent plus présents dans les carrières des femmes.

- Proposer des femmes pour les prix de la SFP.

- Mettre en place un système de mentorat (par une interlocutrice pour aider les femmes lors de leurs évaluations, candidatures et reprises d'activité après un congé de maternité ou de maladie, pour les inciter à demander/accepter des responsabilités...).

- Représenter la SFP à différentes conférences et structures internationales sur l'égalité des genres ("Women in Physics" de l'IUPAP(6) et de l'EPS).

- Aider les femmes à se déplacer pour participer à des conférences (encourager les organisateurs à prévoir des modes de garde d'enfants accessibles aux congressistes et/ou à créer une ligne budgétaire dédiée aux frais de garde ${ }^{(7)}$, suggérer aux employeurs de prendre en compte ces frais dans les frais de mission, mettre en place des bourses pour les femmes pour participer à des congrès internationaux). À notre époque où la Société Française de Physique cherche à élargir son assiette d'adhésion, il est temps qu'elle devienne " la SFP qui rapproche les Hommes et les Femmes de la Science ". Seuls 13\% des adhérent(e)s sont des femmes, cela doit changer!

Véronique Pierron-Bohnes Présidente de la commission IPCMS, BP 43, 67034 Strasbourg Cedex 2 www-ipcms.u-strasbg.fr/spip.php?article42

Si cette action vous intéresse, femme ou homme, rejoignez-nous !

Envoyez un mail à vero@ipcms.unistra.fr 


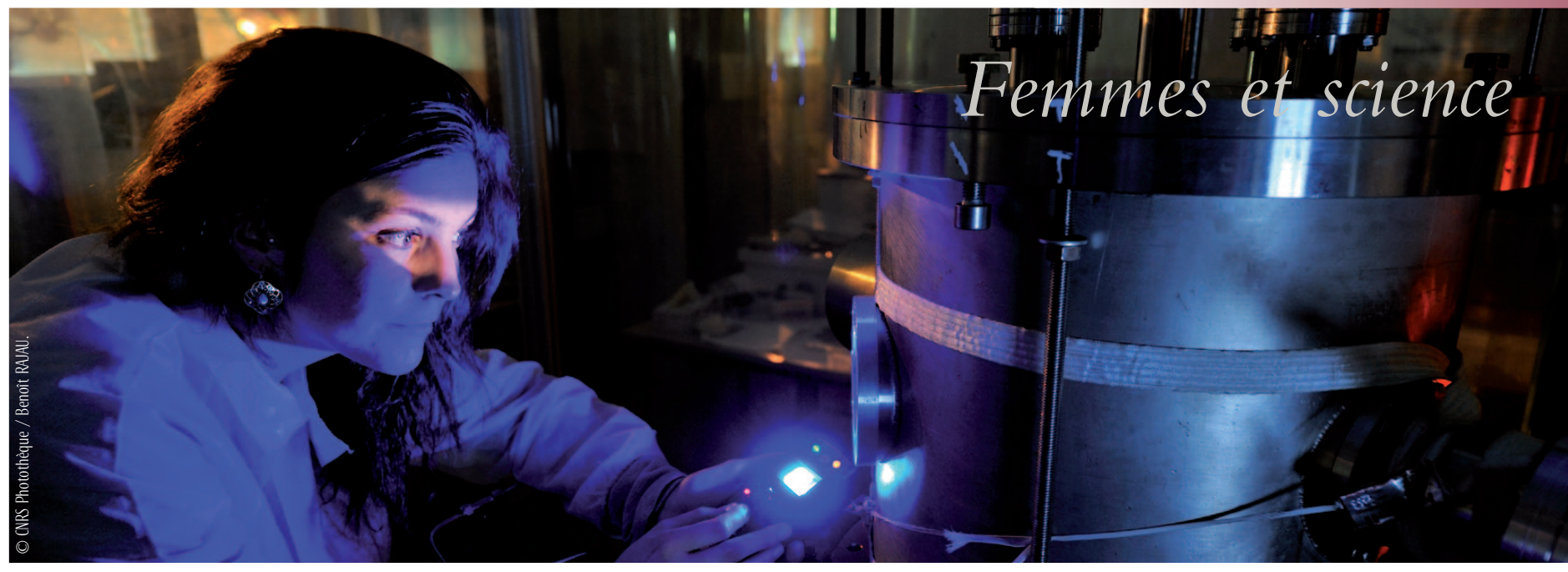

Pourcentages de femmes dans les deux corps de chercheur(se)s pour l'enseignement supérieur, le CNRS et le CEA.

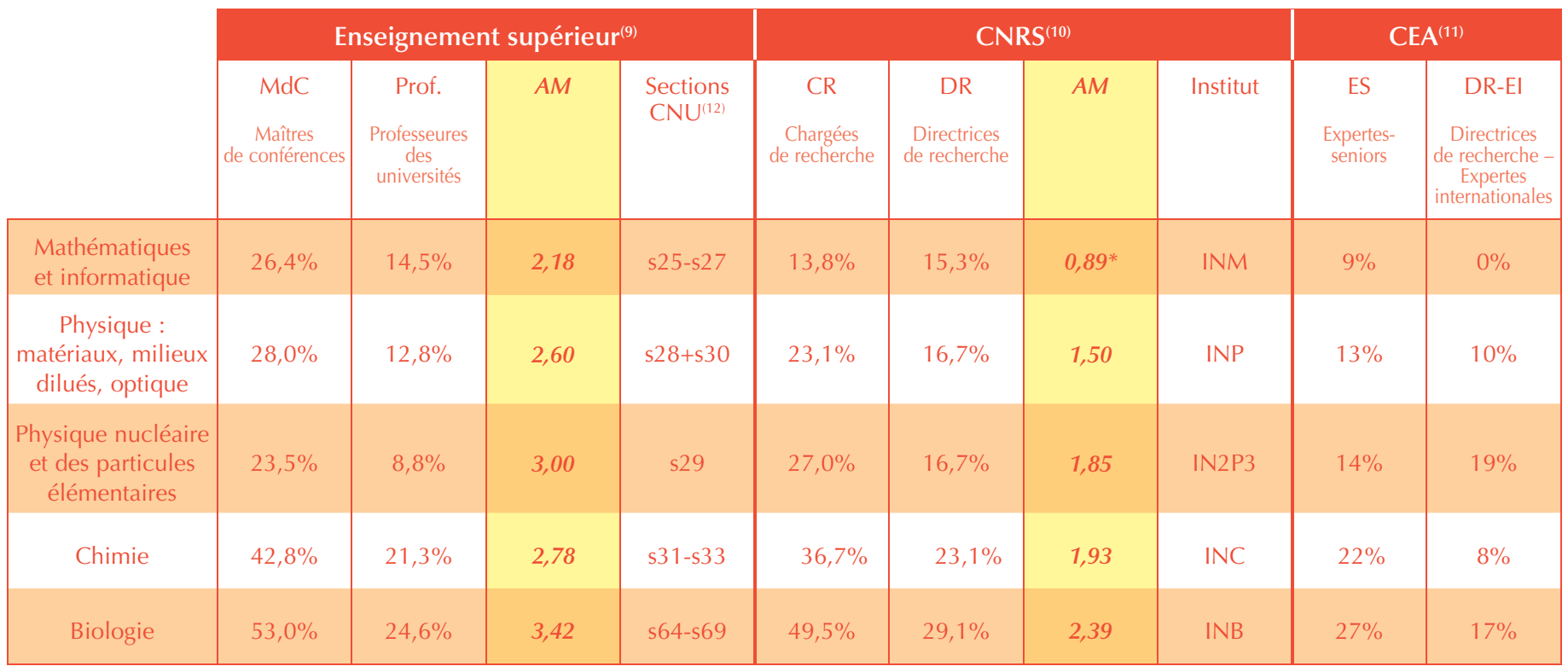

Le pourcentage de filles ${ }^{(8)}$ est de $47 \%$ pour la réussite au baccalauréat $\mathrm{S}$.

AM ou Avantage masculin : rapport des indices de parité (= nombre de femmes/nombre d'hommes) entre les cadres B et A ; plus la valeur de AM est supérieure à 1, plus les femmes ont des difficultés à passer cadre A comparées aux hommes.

* En mathématiques, les chargés de recherche candidatent plus systématiquement comme professeurs que dans les autres spécialités, ce qui explique les chiffres singuliers dans cet institut du CNRS. Il est plus juste de considérer les chiffres globaux CNRS + ES : 26\% de femmes pour le total $B(M d C+C R)$ et $15 \%$ de femmes pour le total $A$ (Prof $+D R$ ), soit $A M=2,05$.

(1) Cette action est complémentaire de celles de "Femmes \& Sciences » www.femmesetsciences.fr/ et www.femmesetsciences.fr/ideesrecues.htm, de "Femmes et mathématiques ": www.femmes-et-maths.fr/,

et de l'Association des femmes ingénieurs : www.femmes-ingenieurs.org/

(2) www.refletsdelaphysique.fr/articles/refdp/pdf/2009/02/refdp200914p24.pdf

(3) Les prix de la SFP ont été très longtemps exclusivement masculins : http://sfp.in2p3.fr/Prix/prix_anciens.html, et sont actuellement timidement féminisés : www.sfpnet.fr/front_office/index.php?rubrique $=$ prix.

(4) Le plafond de verre désigne le fait que, dans une structure hiérarchique, les niveaux supérieurs sont plus difficilement accessibles à certaines catégories de personnes, ici les femmes.

(5) www.sfpnet.fr/front_office/index.php?rubrique $=$ commissions\&id $=106$

(6) International Union of Pure and Applied Physics.

(7) Exemples de conférences qui ont mis en place de telles aides :

- The American Society for Cell Biology, the American Physical Society/ Division of Fluid Dynamics et le réseau d'excellence européen SoftComp : prise en charge des frais occasionnés par la garde des enfants pendant le congrès.
- The Materials Research Society (congrès annuel à Boston depuis 2008) et The Biophysical Society américaine (congrès annuel 2011 à Baltimore) : système de garde en journée sur place.

- The Max Planck Institute for the Physics of Complex Systems à Dresde mise à disposition de logements qui permettent d'accueillir les familles avec enfants avec un système de garde d'enfants fonctionnant en journée. - The Institute of Physics anglais (IOP) a créé un fond pour aider les membres de l'IOP en charge d'autres personnes (parents ou enfants) à participer à leurs meetings.

(8) Source : Filles et garçons sur le chemin de l'égalité de l'école à l'enseignement supérieur: http://media.eduscol.education.fr/file/Valeurs_republicaines/46/0/filles_ garcons_chiffres2010_139460.pdf

(9) Source : Enseignants-chercheurs : répartition par discipline, région et sexe: http://sd-2.archive-host.com/membres/up/38164215153597045/ Stat_CNU_Sexuee_IDF_Regions.pdf

(10) Source : La parité dans les métiers du CNRS 2008/09: www.cnrs.fr/mpdf/IMG/pdf/livretparite20082009_bd.pdf.pdf

(11) Source : A-M. Jonquière, responsable de la filière "Experts " du CEA.

(12) Voir l'intitulé des sections du CNU sur : www.cpcnu.fr/sectionsCnu.htm 